\title{
VARIATIONS OF BETA GLUCURONIDASE CONCENTRATION IN ABNORMAL HUMAN SYNOVIAL FLUID ${ }^{1}$
}

\author{
BY RALPH F. JACOX AND ALEXANDRA FELDMAHN 2 \\ (From the University of Rochester, School of Medicine and Dentistry, Rochester, N. Y.) \\ - (Submitted for publication September 1, 1954; accepted October 27, 1954)
}

Enzyme studies of human synovial fluid have revealed the presence of amylase (1), a protease (2), and lipase (1), and a peroxidase (2). Alkaline phosphatase (3) has also been found and more recently, the enzyme aminotripeptidase (4) has been demonstrated. No studies have been made of the $\beta$ glucuronidase content of human synovial fluid. In view of the significance of the enzyme in mucin metabolism (5) it was felt that an investigation of this problem would be of interest.

The present report represents a study of the $\beta$ glucuronidase concentration of synovial fluids obtained from patients with various pathologic joint diseases. In several instances, serial assays were made in different patients suffering with degenerative joint disease, rheumatoid arthritis, or pyogenic arthritis. It will be shown that significant variations of enzyme concentration are found in these different types of joint disease.

\section{METHOD}

Synovial fluid was obtained from human patients by aspiration of the knee, or elbow joint. The aspirated fluid was placed in a bottle containing sufficient potassium oxalate to delay clot formation. As soon as possible 1.0 millilitre aliquots of this material were pipetted into each of two tests tubes. In one tube, serving as the control, 4.0 millilitres of $0.1 \mathrm{M}$ acetate buffer of $\mathrm{pH} 4.5$ was added. Three and five-tenths millilitres of $0.1 \mathrm{M}$ acetate buffer was added to the second tube. The test tubes were then placed in a $37^{\circ} \mathrm{C}$. waterbath. After a sufficient time had elapsed for temperature equilibration of the solution of joint fluid, 0.5 millilitres of $0.005 \mathrm{M}$ phenolphthalein glucuronide 8 was added to the second tube. A few drops of chloroform were added to each tube to inhibit bacterial growth. Both tubes were incubated in

\footnotetext{
1 Research carried out under a grant for the study of rheumatic fever from the Masonic Foundation for Medical Research and Human Welfare.

2 Research Fellow in Medicine, University of Rochester School of Medicine and Dentistry.

3 Obtained from the Sigma Chemical Co., 4648 Easton Avenue, St. Louis 13, Missouri.
}

the $37^{\circ} \mathrm{C}$. waterbath for 16 to 24 hours. At the conclusion of the incubation period, 5.0 millilitres of $0.1 \mathrm{M}$ glycine buffer of $\mathrm{pH} 10.5$ was pipetted into each tube. Both were rapidly centrifuged to sediment particulate material. The supernatant fluid was then removed and assayed for phenolphthalein concentration. A Lumetron colorimeter with a 540 filter. was employed, with the colorimeter set for the corresponding blank of each fluid to be analyzed. By interpolation from a previously prepared standard curve, the concentration of phenolphthalein. was then calculated. The concentration of $\boldsymbol{\beta}$ glucuronidase was then expressed as one microgram of phenolphthalein liberated per millilitre per hour equal to one unit. This method of glucuronidase assay is a modification of that described by Talalay, Fishman, and Huggins (6).

\section{RESULTS}

Fifty-six patients with various pathologic joint disorders have been studied. In some instances serial determinations have been made on the same patient over a period of several months. The experimental results obtained from all assays of joint fluid are shown in Figure 1. It will be observed that patients with severe rheumatoid arthritis had considerable $\beta$ glucuronidase in the synovial fluid while those that had a mild and slowly progressive rheumatoid arthritis had less $\beta$ glucuronidase. In contrast, the data obtained from patients with degenerative joint disease show that with a single exception, these synovial fluids had significantly less $\beta$ glucuronidase than any of the patients with rheumatoid arthritis.

Three patients with pyogenic arthritis were studied. It will be noted that the enzyme concentration in these individuals was greatly elevated, rising to values a thousand times those seen in degenerative joint disease. In patients with other types of joint disease (Figure 1), it will be observed that one individual with gout No. 5 , one with psoriasis and arthritis No. 1, and one with a Charcot joint No. 6 had values somewhat higher than observed in patients with degenerative joint disease. Two patients with a traumatic joint ef- 
fusion, T, one with uremia No. 2 and one with an unexplained joint effusion No. 4 had low concentrations of $\beta$ glucuronidase. One patient with a proliferative synovitis No. 3 also had a low concentration of synovial fluid $\beta$ glucuronidase.

Data on three patients on whom serial determinations of glucuronidase were made are shown in Figure 2. The first patient was a 72-year-old man admitted to the Municipal Hospital with an acute febrile illness characterized by mild delirium, an acute monoarticular arthritis, and cutaneous lesions suggestive of meningococcal bacteremia. Cerebrospinal fluid examination was negative and several blood cultures showed no growth of bacteria. The left elbow was swollen, red and painful and there was marked restriction of joint motion. An aspiration of the joint revealed purulent fluid, which contained $123,000 \mathrm{~W}$. B. C. per mm. ${ }^{3}$, most of which were polymorphonuclear leucocytes. This fluid showed no bacterial growth after culture aerobically, anaerobically, and under $\mathrm{CO}_{2}$. After therapy for several days with penicillin, a gradual fall of fever and a slow subsidence of joint swelling and improvement of joint motion occurred. However, the patient developed a severe, exfoliative dermatitis due to penicillin sensitivity. During this reaction, the left knee became swollen, red and tender. An aspiration of fluid from this joint revealed a cell count of 5,600 W. B. C. per mm. ${ }^{8}$ After ACTH therapy, both the dermatitis and arthritis improved. On cessation of ACTH therapy, a recrudescence of joint swelling involving both knees, and wrists, and left elbow occurred.

While the etiology of the arthritis in this patient is not unequivocally defined, it was felt that the patient had an acute bacterial infection, possibly due to a meningococcus, which caused a suppurative arthritis of the left elbow. Subsequently this disease developed into multi-articular involvement which was more characteristic of a rheumatoid arthritis.

$\beta$ glucuronidase studies of the elbow joint fluid aspirations (indicated by the solid circle, Figure 2) revealed very high levels of enzyme activity. After chemotherapy, a decrease in enzyme concentration was found. With later recrudescence of joint swelling, an increase of $\beta$ glucuronidase was observed.
In the second patient, the data for $\beta$ glucuronidase determinations are illustrated successively in a 68-year-old woman with an acute, severe rheumatoid arthritis. Initially this patient had little relief with systemic cortisone therapy or by local instillation of Hydrocortone ${ }^{\circledR}$ into both knee joints. Three months before her first clinic visit, and shortly after she sustained a hip fracture, the patient developed multi-articular pain, swelling and increased limitation of joint function. During the next six months there was progressive, severe acute joint disease involving both knees and the left wrist.

The first observation of the $\beta$ glucuronidase concentration of the knee fluid (indicated by open circles, Figure 2) was made in July, 1953. At this time $50 \mathrm{mgm}$. of cortisone, administered by mouth, afforded moderate relief of joint symptoms. However, the patient required occasional supplementary injections of compound $\mathrm{F}$ into the left knee during August. In September, 1953, the patient developed more pain and effusion of the

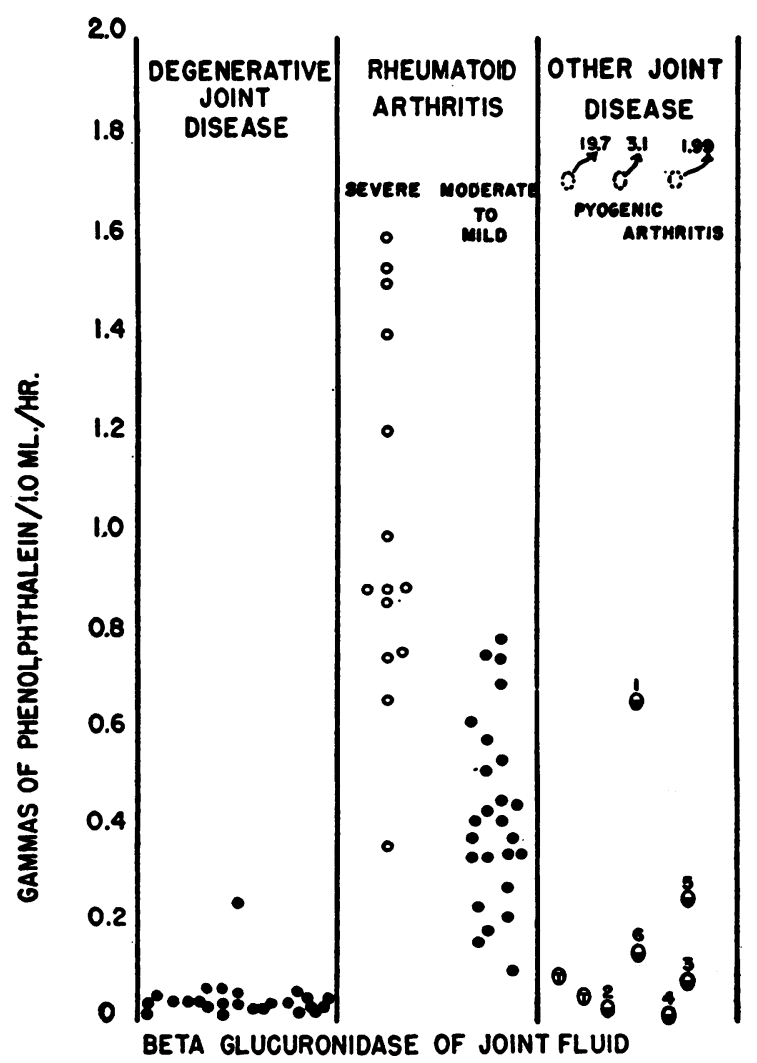

Fig. 1. Synovial Fluid Concentration of $\beta$ Glucuronidase Found in Various Types of Joint Disease 
wrist and knees and other joints became painful and swollen. After increasing the cortisone dosage to 75 mgm., some improvement resulted, but her symptoms again increased in November. In November, Butazolidin ${ }^{\circledR}$ therapy was instituted at a time when cortisone was being gradually reduced. This therapeutic change produced more joint pain and disability. In early December, 50 mgm. of cortisone each day was re-instituted and Butazolidin ${ }^{\otimes}$ discontinued. By the end of December, while still taking $50 \mathrm{mgm}$. of cortisone each day, the patient began to improve. She was less depressed and all the previously swollen and tender joints, excepting the right knee, were less painful and swollen. By January, 1954, she was better than she had been in months.

The $\beta$ glucuronidase determinations made periodically throughout this six-month interval reflected the severity of the arthritic process. During September (65th day of observation, Figure 2 ), when the peak of clinical activity was greatest, the highest concentration of enzyme was found. This was followed by a decrease in $\beta$ glucuronidase to a value of 0.42 units during January, 1954 (180th day-Figure 2) when clinical improvement was evident.

The third patient is a 64-year-old woman with severe bilateral, degenerative joint disease involving both knees. She had considerable symptomatic relief of pain with monthly injections of Hydrocortone ${ }^{(\$)}$ into the synovial space of each knee. It will be noted that the $\beta$ glucuronidase concentration in the synovial fluid of this patient was considerably less than in the patient with rheumatoid arthritis. The enzyme remained consistently low during the period of observation (lower curve, Figure 2).

These data suggest that the concentration of $\beta$ glucuronidase reflects the severity of inflammation within the synovium and joint space. In patients with septic arthritis the enzyme is greatly increased. In patients with degenerative joint disease or traumatic effusion, low concentrations are found. The rheumatoid arthritic patients were divided into mild and severe forms of disease by evaluation of the duration and degree of symptoms of active inflammation, degree of progressive joint destruction and by determination of the erythro-

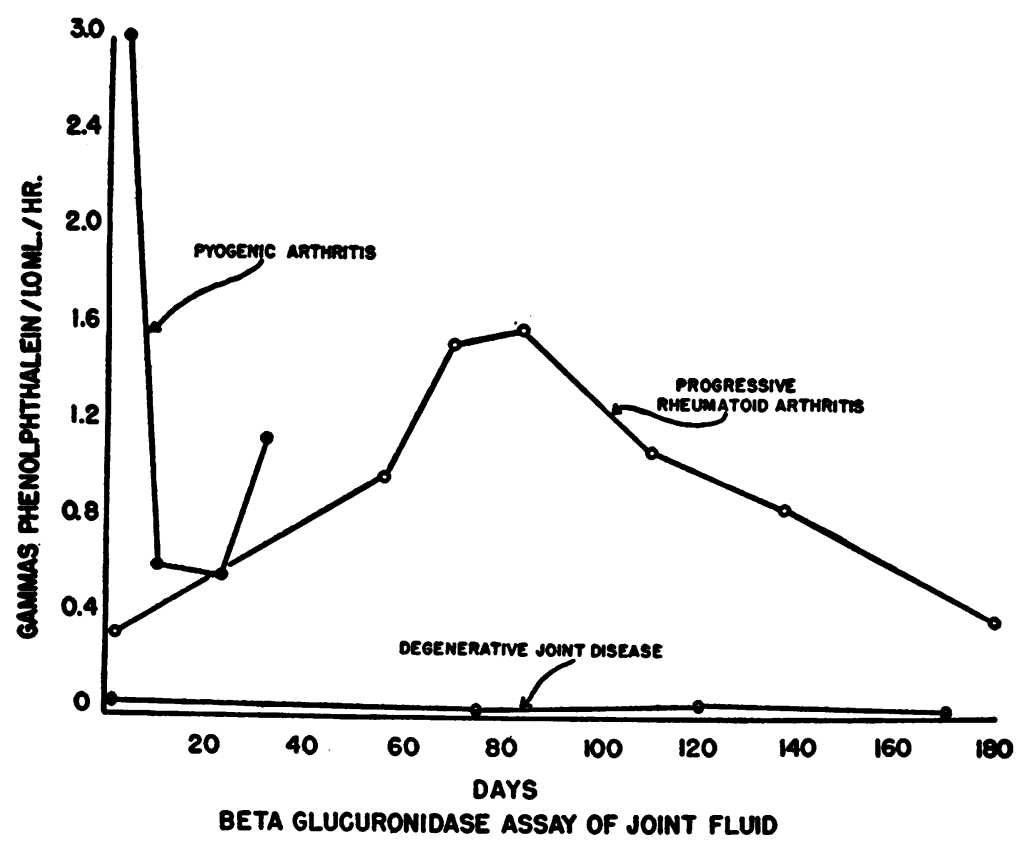

Fig. 2. Results of Successive Determinations of $\beta$ Glucuronidase Concentration in the Synovial Fluids of Patients with A) Pyogenic Arthritis (Solid Circle), B) Rheumatoid Arthritis (Open Circle), and C) Degenerative Joint Disease (Semi-Closed Circle) 
cyte sedimentation rate, plasma alpha globulin and fibrinogen concentrations, degree of anemia and extent of radiographic bone and joint alteration. With such a clinical estimation it was observed that higher concentrations of synovial fluid $\beta$ glucuronidase are usually found in the patient with severe rheumatoid arthritis than in the mild, subacute disease (Figure 1).

A comparison of $\beta$ glucuronidase concentration with the leucocyte count of joint fluid revealed some correlation. If the synovial fluid leucocyte count was very elevated, high concentrations of enzyme were found. If a few leucocytes were present, only small amounts of $\beta$ glucuronidase were found. However, no absolute linear relationship was observed.

Joint fluid obtained from patients with severe rheumatoid arthritis and with septic arthritis was centrifuged to remove nearly all the formed elements. The supernatant fluid was then analyzed for $\beta$ glucuronidase activity. In one instance, the untreated joint fluid had a $\beta$ glucuronidase activity of 3.09 units. The centrifuged supernatant fluid had an activity of 0.76 units or nearly ten times the concentration found in synovial fluid of degenerative joint disease. In another patient with a septic arthritis containing 216,000 polymorphonuclear leucocytes, the $\beta$ glucuronidase activity was 19.7 units, while the centrifuged supernatant fluid assayed 6.15 units.

It thus appears that enzyme, apart from that contributed by leucocytes, may accumulate in synovial fluid. This soluble enzyme is probably derived from disintegrated leucocytes, although contributions by synovial secretion cannot be excluded.

\section{DISCUSSION}

The enzyme $\beta$ glucuronidase, which splits conjugated glucuronides, is widely distributed within the human body (5). It is found in high concentration in liver and spleen and in lower concentrations in other tissues. Polymorphonuclear leucocytes contain $\beta$ glucuronidase while lymphocytes, platelets, and red blood corpuscles contain none (5). Pleural and ascitic effusions due to various pathologic disorders have been shown to contain $\beta$ glucuronidase (5). This enzyme is also found in tears, and in gastric and bronchial secretions (5).

The present investigation reveals that synovial fluid obtained from human patients with various joint disorders also contains $\beta$ glucuronidase. Individuals with degenerative joint disease and those with traumatic effusions or unexplained effusions had enzyme concentrations ranging between 0.015 to $\mathbf{0 . 0 8 0}$ units. Only one exception outside of this range was observed. Patients with rheumatoid arthritis had values ranging from 0.190 to 1.0 units. In general, those patients with the most severe rheumatoid arthritis had the highest values within this range, whereas the mild rheumatoid patients had concentrations of $\beta$ glucuronidase in the lower part of this range. Patients with septic arthritis had concentrations of $\beta$ glucuronidase as high as 19.7 units.

The concentration of $\beta$ glucuronidase in synovial fluid appears to reflect the cellular response to the inflammatory change in the synovial membrane. Not only is the enzyme contained within the leucocytes, but it is also found in the cell-free fluid. Thus the contribution of intact leucocytes, disintegrated leucocytes and possible contribution by synovial membrane structures is measured when unclotted synovial fluid is assayed for enzyme activity.

The data from this investigation suggest that the estimation of $\beta$ glucuronidase in synovial fluid may afford another method of estimating severity of inflammatory change within the joint structure and may be of some aid in the differentiation of early rheumatoid arthritis from non-inflammatory joint disease.

\section{SUM MARY}

$\beta$ glucuronidase has been demonstrated in human synovial fluid. In pathological states such as rheumatoid arthritis and pyogenic arthritis, there is as much as 200 to 1000 -fold increase of enzyme over that found in synovial fluid from patients with degenerative joint disease or those with a traumatic joint effusion. While much of the $\beta$ glucuronidase of the synovial fluid is contributed by the polymorphonuclear leucocytes, some of it is soluble. The soluble fraction may be derived from disintegrated leucocytes or from secretion by the synovial membrane. 


\section{REFERENCES}

1. Podkaminsky, N. A., Rôle de ferments du liquide synovial. Compt. rend Soc. de biol., 1931, 106, 915.

2. Ropes, M. W., and Bauer, W., Synovial Fluid Changes in Joint Disease. Cambridge, Mass., Commonwealth Fund, 1953, p. 9.

3. Ropes, M. W., Bennett, G. A., and Bauer, W., The origin and nature of normal synovial fluid. $\mathrm{J}$. Clin. Invest., 1939, 18, 351.

4. Ziff, M., Scull, E., Ford, D., McEwen, C., and Bunim,
J. J., Effects of intra-articular hydrocortisone acetate on the clinical course, aminotripeptidase activity and other changes in synovial fluid in rheumatoid arthritis. Ann. Rheumat. Dis., 1952, 11, 301.

5. Fishman, W. H., $\beta$-glucuronidase. The Enzymes, Chemistry and Mechanism of Action. New York, Academic Press Inc., 1950, vol. 1, part 1, p. 635.

6. Talalay, P., Fishman, W. H., and Huggins, C., Chromogenic substances. II. Phenolphthalein glucuronic acid as substrate for the assay of glucuronidase activity. J. Biol. Chem., 1946, 166. 757. 